\title{
A VARIANT OF THE BARBAN-DAVENPORT-HALBERSTAM THEOREM
}

\author{
ETHAN SMITH
}

\begin{abstract}
Let $L / K$ be a Galois extension of number fields. The problem of counting the number of prime ideals $\mathfrak{p}$ of $K$ with fixed Frobenius class in $\operatorname{Gal}(L / K)$ and norm satisfying a congruence condition is considered. We show that the square of the error term arising from the Chebotarëv Density Theorem for this problem is small "on average." The result may be viewed as a variation on the classical Barban-Davenport-Halberstam Theorem.
\end{abstract}

\section{INTRODUCTION}

The Barban-Davenport-Halberstam Theorem [1, 2, 3] concerns the average square error for the Prime Number Theorem for primes in arithmetic progressions. In particular, if we let

$$
\theta(x ; q, a):=\sum_{\substack{p \leq x \\ p \equiv a}} \log p,
$$

then the Barban-Davenport-Halberstam Theorem states that, for every fixed $M>0$,

$$
\sum_{q \leq Q} \sum_{\substack{a=1 \\(a, q)=1}}^{q}\left(\theta(x ; q, a)-\frac{x}{\varphi(q)}\right)^{2} \ll x Q \log x,
$$

provided that $x(\log x)^{-M} \leq Q \leq x$. See [4, p. 169] for a statement of this theorem with $\theta(x ; q, a)$ replaced by the Chebychev function $\psi(x ; q, a)$.

If one adopts the point of view that the Prime Number Theorem for primes in arithmetic progressions is a specialization of the Chebotarëv Density Theorem [9, p. 143] for the cyclotomic extensions $\mathbb{Q}\left(\zeta_{q}\right) / \mathbb{Q}$, then, in light of the Kronecker-Weber Theorem [11, p. 210], the Barban-Davenport-Halberstam Theorem may be interpreted as giving a bound for the average square error for the Chebotarëv Density Theorem taken over all Abelian extensions of $\mathbb{Q}$. It would be natural then to study averages that include non-Abelian extensions as well. In this paper, we consider averages where the Galois group varies over groups of the form $G_{1} \times G_{2}$, where $G_{1}$ is a fixed (potentially non-Abelian) group and $G_{2} \subseteq(\mathbb{Z} / q \mathbb{Z})^{*}$ with $q$ varying.

This work is carried out for the purpose of providing a tool to study the "average LangTrotter" problem in number fields. Much is owed to the related paper of Murty and Murty [15].

2000 Mathematics Subject Classification. 11R44, 11N05, 11N36.

Key words and phrases. Barban-Davenport-Halberstam Theorem, Chebotarëv Density Theorem, large sieve. 


\section{Statement of Results}

Let $L / K$ be a Galois extension of number fields with group $G$, and let $C$ be a fixed conjugacy class in $G$. For any pair of integers $q$ and $a$, we define

$$
\theta(x ; C, q, a):=\sum_{\substack{\mathbf{N p} \leq x \\\left(\frac{L / K}{\mathfrak{p}}\right)=C \\ \mathbf{N} \mathfrak{p} \equiv a(\bmod q)}} \log \mathbf{N p},
$$

where the sum is taken over all finite primes $\mathfrak{p}$ of $K$ that do not ramify in $L, \mathbf{N}=\mathbf{N}_{K / \mathbb{Q}}$ denotes the norm, and $\left(\frac{L / K}{\mathfrak{p}}\right)$ denotes the Frobenius class of $\mathfrak{p}$ in $G$.

For each positive integer $q$, let $\zeta_{q}$ denote a primitive $q$-th root of unity. If $L \cap K\left(\zeta_{q}\right)=K$, then

$$
\mathcal{G}_{q}:=\operatorname{Gal}\left(L\left(\zeta_{q}\right) / K\right) \cong \operatorname{Gal}\left(K\left(\zeta_{q}\right) / K\right) \times G .
$$

See [12, p. 267]. As in [20], there is a natural composition of maps

$$
\operatorname{Gal}\left(K\left(\zeta_{q}\right) / K\right) \longleftrightarrow \operatorname{Gal}\left(\mathbb{Q}\left(\zeta_{q}\right) / \mathbb{Q}\right) \longrightarrow(\mathbb{Z} / q \mathbb{Z})^{*}
$$

Letting $G_{K, q}$ denote the image of this map and $\varphi_{K}(q):=\left|G_{K, q}\right|$, the Chebotarëv Density Theorem gives the asymptotic identity

$$
\theta(x ; C, q, a) \sim \frac{1}{\varphi_{K}(q)} \frac{|C|}{|G|} x
$$

as $x \rightarrow \infty$, provided that $a \in G_{K, q}$ and $L \cap K\left(\zeta_{q}\right)=K$. We remark that the Frobenius class of $\mathfrak{p}$ in $\operatorname{Gal}\left(K\left(\zeta_{q}\right) / K\right)$ is determined by the residue of $\mathbf{N p}$ modulo $q$.

In this paper, we establish a variant of the classical Barban-Davenport-Halberstam Theorem similar to the variant of the classical Bombieri-Vinogradov Theorem proved by Murty and Murty in [15]. That is, we show an upper bound for the average square error in the asymptotic (2) when averaging over the set of all $q$ such that $L \cap K\left(\zeta_{q}\right)=K$ and over all $a \in G_{K, q}$. More precisely, we prove the following result.

Theorem 1. Let $M>0$. If $x(\log x)^{-M} \leq Q \leq x$, then

$$
\sum_{q \leq Q}^{\prime} \sum_{a \in G_{K, q}}\left(\theta(x ; C, q, a)-\frac{1}{\varphi_{K}(q)} \frac{|C|}{|G|} x\right)^{2} \ll x Q \log x,
$$

where the prime on the outer summation indicates that the sum is to be restricted to those $q \leq Q$ satisfying $L \cap K\left(\zeta_{q}\right)=K$. The constant implied by the symbol $\ll$ depends on $L$ and $M$.

Remark. By the triangle inequality, Theorem 1 holds in the case that $C$ is a union of conjugacy classes in $G=\operatorname{Gal}(L / K)$ as well.

Remark. The main result of [15] is precisely the Bombieri-Vinogradov type analogue of Theorem 1 in the case $K=\mathbb{Q}$. M. R. Murty and K. Petersen have recently extended the main result of [15] to the setting of number fields. However, their average on $q$ is restricted to $q$ satisfying $L \cap \mathbb{Q}\left(\zeta_{q}\right)=\mathbb{Q}$. The author is grateful for receiving a copy of their preprint [17]. 
As mentioned in the introduction, this work is carried out for the purpose of providing a tool to study the average Lang-Trotter problem in number fields. In particular, it useful for extending the works [5, 7] to the "totally non-Abelian" setting. As another application, we give the following.

Theorem 2. Suppose that $K=\mathbb{Q}$ and that $L / \mathbb{Q}$ is a totally non-Abelian Galois extension (i.e., $L \cap \mathbb{Q}\left(\zeta_{q}\right)=\mathbb{Q}$ for all $q \geq 1$ ). There exist constants $c, c^{\prime}$ so that, for each fixed $M>0$,

$$
\sum_{q \leq x} \sum_{\substack{a=1 \\(a, q)=1}}^{q}\left(\theta(x ; C, q, a)-\frac{|C|}{\varphi(q)|G|} x\right)^{2}=\frac{|C|}{|G|} x^{2} \log x+c x^{2}+O\left(\frac{x^{2}}{(\log x)^{M}}\right) ;
$$

and if $1 \leq Q \leq x$,

$$
\begin{aligned}
\sum_{q \leq Q} \sum_{\substack{a=1 \\
(a, q)=1}}^{q}\left(\theta(x ; C, q, a)-\frac{|C|}{\varphi(q)|G|} x\right)^{2}= & \frac{|C|}{|G|} x Q \log x-\frac{|C|^{2}}{|G|^{2}} x Q \log (x / Q)+c^{\prime} x Q \\
& +O\left(Q^{\frac{5}{4}} x^{\frac{3}{4}}\right)+O\left(\frac{x^{2}}{(\log x)^{M}}\right) .
\end{aligned}
$$

Remark. See [21] for a version of this result when $K=L$ and $K$ is not necessarily equal to Q.

We omit the proof of Theorem 2 since it follows from Theorem 1 by adapting the techniques of Hooley [8, pp. 209-212] in the obvious manner. It would be natural to try to remove the restrictions on $K$ and $L$ from the statement of Theorem 2 . However, if $K \neq \mathbb{Q}$, then one seems to be left with the task of counting the number of prime ideals of a given norm and fixed Frobenius class. In the case, that $L$ is not linearly disjoint from every cyclotomic extension, Theorem 1 requires that we remove the "offending moduli" from the outer sum over $q$. The result is that Hooley's trick (see [8, p. 210]) of "switching divisors" does not seem to work anymore.

The remainder of the article is outlined as follows. In Section 3, we recall some useful facts about characters and $L$-functions. Section 4 is devoted to the proof of Siegel-Walfisz type results. The final section is concerned with the proof of Theorem 1 ,

\section{Class functions, characters, and Artin $L$-Functions}

In this section, we set up some general notation and recall some of the necessary background information concerning Galois representations and Artin $L$-functions. For a number field $F$, we will write $d_{F}$ for the discriminant of $F$. If $L / F$ is an extension of number fields, we will write $n_{L / F}:=[L: F]$ for the degree of the extension. In the case that the base field is $\mathbb{Q}$, we will simply write $n_{L}:=[L: \mathbb{Q}]$.

Suppose that $L / F$ is a Galois extension of number fields, say with group $G$. Consider a group representation $\rho: G \rightarrow \mathrm{GL}_{n}(\mathbb{C})$. The character associated to $\rho$ is the function $\eta=\eta_{\rho}$ defined by $\eta(\sigma):=\operatorname{Tr}(\rho(\sigma))$. For each finite prime $\mathfrak{p}$ of $F$ unramified in $L$, choose a prime $\mathfrak{P} \mid \mathfrak{p}$ in $L$, and let $\sigma_{\mathfrak{P}}$ denote the Frobenius at $\mathfrak{P}$. Further, put

$$
L_{\mathfrak{p}}(s, \eta):=\operatorname{det}\left(I-\rho\left(\sigma_{\mathfrak{P}}\right) \mathbf{N p}^{-s}\right)^{-1}
$$

and note that this definition does not depend on the choice of $\mathfrak{P} \mid \mathfrak{p}$. For the ramified primes, there is a similar but slightly more complicated definition for $L_{\mathfrak{p}}(s, \eta)$, and we refer the reader 
to [14, pp. 8-9] or [16, p. 27] for the details. For $\operatorname{Re}(s)>1$, the Artin $L$-series associated to $\rho$ or $\eta$ is defined by the Euler product

$$
L(s, \eta):=\prod_{\mathfrak{p}} L_{\mathfrak{p}}(s, \eta),
$$

where the product is over all the prime ideals $\mathfrak{p}$ of $F$ (including the ramified primes).

Associated to this $L$-series is an ideal of $F$ called the Artin conductor. We denote this ideal by $\mathfrak{f}(\eta, L / F)$. It is defined in terms of higher ramification groups, and we refer the reader to [14, pp. 13-14] or [16, p. 28] for more detail. The conductor of the $L$-series is then defined by

$$
A(\eta):=\left|d_{F}\right|^{\eta(1)} \mathbf{N}_{F / \mathbb{Q}}(\mathfrak{f}(\eta, L / F)) .
$$

A function $\xi: G \rightarrow \mathbb{C}$ satisfying $\xi\left(g^{-1} \sigma g\right)=\xi(\sigma)$ for all $g \in G$ is said to be a class function on $G$. Note that the character of a group representation is a class function. Given any class function $\xi$ on $G$, we define the weighted prime counting function

$$
\theta(x ; L / F, \xi):=\sum_{\mathbf{N} \mathfrak{p} \leq x} \xi\left(\sigma_{\mathfrak{p}}\right) \log \mathbf{N p}
$$

where the sum is over the prime ideals of $F$ that do not ramify in $L$, and $\sigma_{\mathfrak{p}}$ denotes any element of the Frobenius class at $\mathfrak{p}$. We also define the function

$$
\psi(x ; L / F, \xi):=\sum_{\mathbf{N p}^{m} \leq x} \xi\left(\sigma_{\mathfrak{p}}^{m}\right) \log \mathbf{N p}
$$

where now we include all powers of prime ideals of $F$ that do not ramify in $L$. For a prime $\mathfrak{p}$ of $F$ ramified in $L$ and $\mathfrak{P}$ a prime of $L$ lying above $\mathfrak{p}$, let $D_{\mathfrak{P}}$ and $I_{\mathfrak{P}}$ denote the decomposition and inertia group at $\mathfrak{P}$ respectively. We extend the definition of $\xi$ to ramfied $\mathfrak{p}$ by

$$
\xi\left(\sigma_{\mathfrak{p}}^{m}\right):=\frac{1}{\left|I_{\mathfrak{P}}\right|} \sum_{g} \xi(g),
$$

where the sum is over all $g \in D_{\mathfrak{P}}$ such that $g \equiv \sigma_{\mathfrak{P}}^{m}\left(\bmod I_{\mathfrak{P}}\right)$. We now define

$$
\widetilde{\psi}(x ; L / F, \xi):=\sum_{\mathbf{N p}^{m} \leq x} \xi\left(\sigma_{\mathfrak{p}}^{m}\right) \log \mathbf{N p},
$$

where the sum is over all powers of prime ideals $\mathfrak{p}^{m}$ of $F$ including the ramified ones. We will sometimes suppress the field extension in the notation for these functions when there is no danger of confusion.

It will sometimes be necessary to switch between $\theta$ and $\widetilde{\psi}$. The difference between these functions is

$$
\theta(x ; \xi)-\widetilde{\psi}(x ; \xi)=\sum_{\substack{\mathbf{N} \mathfrak{p}^{m} \leq x, m \geq 2}} \xi\left(\sigma_{\mathfrak{p}}^{m}\right) \log \mathbf{N p}+\sum_{\substack{\mathbf{N} \mathfrak{p} \leq x \\ \mathfrak{p} \text { ram. }}} \xi\left(\sigma_{\mathfrak{p}}\right) \log \mathbf{N p} .
$$

The first sum on the right is over all prime ideals $\mathfrak{p}$ of $F$ and all integers $m \geq 2$, and is trivially bounded by $\|\xi\| n_{F} \sqrt{x} \log x$, where $\|\xi\|:=\max _{g \in G} \xi(g)$. The second sum on the right is over only those prime ideals $\mathfrak{p}$ of $F$ which ramify in $L$. For the second sum, we have 
the bound

$$
\left|\sum_{\substack{\mathfrak{N} \mathfrak{p} \leq x \\ \mathfrak{p} \text { ram. }}} \xi\left(\sigma_{\mathfrak{p}}\right) \log \mathbf{N} \mathfrak{p}\right| \leq\|\xi\| \sum_{\mathfrak{p} \mid \mathfrak{d}(L / F)} \log \mathbf{N} \mathfrak{p} \leq \frac{2\|\xi\|}{n_{L / F}} \log \left|d_{L}\right|
$$

by [19, Prop. 5]. Therefore,

$$
|\widetilde{\psi}(x ; \xi)-\theta(x ; \xi)| \leq \| \xi|| n_{F}\left(\sqrt{x} \log x+\frac{2}{n_{L}} \log \left|d_{L}\right|\right) .
$$

Any class function $\xi$ can be written as

$$
\xi=\sum_{\eta} a_{\eta} \eta
$$

where $a_{\eta} \in \mathbb{C}$ and $\eta$ ranges over the irreducible characters of $G$. In particular, if $C$ is a conjugacy class of $G$ and $g_{C} \in C$, then the characteristic function of $C$ may be decomposed as

$$
\delta_{C}=\frac{|C|}{|G|} \sum_{\eta} \bar{\eta}\left(g_{C}\right) \eta
$$

In general, we have the identity

$$
\widetilde{\psi}(x ; L / F, \xi)=-\frac{1}{2 \pi i} \sum_{\eta} a_{\eta} \int_{(2)} \frac{L^{\prime}(s, \eta)}{L(s, \eta)} \frac{x^{s}}{s} d s,
$$

where the line of integration is $\operatorname{Re}(s)=2$.

Now, suppose that $H$ is a subgroup of $G=\operatorname{Gal}(L / F)$ and $E=L^{H}$ is the fixed field of $H$. If $\xi$ is a class function on $H$, we may define the induced class function $\operatorname{Ind}_{H}^{G}(\xi)$ as follows. First extend $\xi$ to all of $G$ by $\xi(\sigma)=0$ for all $\sigma \in G \backslash H$; then set

$$
\operatorname{Ind}_{H}^{G}(\xi)(\sigma):=\frac{1}{|H|} \sum_{g \in G} \xi\left(g^{-1} \sigma g\right) .
$$

An important feature of Artin $L$-functions is their invariance under induction. That is, if $\eta$ is a character of $H$, then

$$
L(s, L / E, \eta)=L\left(s, L / F, \operatorname{Ind}_{H}^{G}(\eta)\right) .
$$

See [14, p. 9] for example. Thus, it follows that if $\xi$ is a class function on $H$, then

$$
\widetilde{\psi}(x ; L / E, \xi)=\widetilde{\psi}\left(x ; L / F, \operatorname{Ind}_{H}^{G}(\xi)\right) .
$$

Now suppose that $G=G_{1} \times G_{2}$, and let $\rho_{1}, \rho_{2}$ define irreducible representations of $G_{1}$ and $G_{2}$ respectively. Then the tensor product representation, defined by

$$
\left(\rho_{1} \otimes \rho_{2}\right)\left(g_{1}, g_{2}\right):=\rho_{1}\left(g_{1}\right) \otimes \rho_{2}\left(g_{2}\right),
$$

is an irreducible representation of $G$. Moreover, every irreducible representation of $G$ arises in this way. If $\eta_{1}, \eta_{2}$ are the characters associated to $\rho_{1}, \rho_{2}$ respectively, then the character of the tensor product representation is given by

$$
\left(\eta_{1} \otimes \eta_{2}\right)\left(g_{1}, g_{2}\right)=\eta_{1}\left(g_{1}\right) \eta_{2}\left(g_{2}\right)
$$

See [18, p. 26-28]. Now, suppose $L_{1} / F$ and $L_{2} / F$ are Galois extensions of number fields with $\operatorname{Gal}\left(L_{1} / F\right)=G_{1}$ and $\operatorname{Gal}\left(L_{2} / F\right)=G_{2}$. Suppose further that $L_{1} \cap L_{2}=F$, and set 
$L=L_{1} L_{2}$. Then $L / F$ is Galois with group $G_{1} \times G_{2}$ [12, p. 267]. Finally, we note that the Artin conductors satisfy the relationship

$$
\mathfrak{f}\left(\eta_{1} \otimes \eta_{2}, L / F\right) \mid \mathfrak{f}\left(\eta_{1}, L_{1} / F\right)^{\eta_{2}(1)} \mathfrak{f}\left(\eta_{2}, L_{2} / F\right)^{\eta_{1}(1)}
$$

See [14, p. 80] for example.

\section{Siegel-Walfisz type estimates}

In this section we assume that $L / E$ is an Abelian extension of number fields. It follows that $L\left(\zeta_{q}\right) / E$ is also an Abelian extension. In fact, $\operatorname{Gal}\left(L\left(\zeta_{q}\right) / E\right) \hookrightarrow \operatorname{Gal}(L / E) \times(\mathbb{Z} / q \mathbb{Z})^{*}$. See $[12$, p. 267] for example. By the discussion in the last paragraph of Section 3, any irreducible character $\eta$ of $\operatorname{Gal}\left(L\left(\zeta_{q}\right) / E\right)$ arises as $\eta=\omega \otimes \chi$, where $\omega$ is an irreducible character of $\operatorname{Gal}(L / E)$ and $\chi$ is a Dirichlet character modulo $q$. By (9), we have the following conductor relation

$$
\begin{aligned}
A\left(\omega \otimes \chi, L\left(\zeta_{q}\right) / E\right) & \leq\left|d_{E}\right| \mathbf{N}_{E / \mathbb{Q}}(\mathfrak{f}(\omega, L / E)) \mathbf{N}_{E / \mathbb{Q}}\left(\mathfrak{f}\left(\chi, E\left(\zeta_{q}\right) / E\right)\right) \\
& \leq A(\omega, L / E) q^{n_{E}} .
\end{aligned}
$$

The first objective of this section is to prove the following proposition, which is a generalization of Lemma 6.2 of [15, p. 267].

Proposition 1. Let $L / E$ be an Abelian extension of number fields, $\omega$ an irreducible character of $\operatorname{Gal}(L / E)$, and let $1<q, N$ be any integers so that $A(\omega) q^{n_{E}} \leq(\log x)^{N}$. Then there exists a positive constant $c_{0}(E, N)$ so that, for any Dirichlet character $\chi$ modulo $q$ such that $\omega \otimes \chi$ is not trivial,

$$
\widetilde{\psi}\left(x ; L\left(\zeta_{q}\right) / E, \omega \otimes \chi\right) \ll(\log A(\omega \otimes \chi)) x \exp \left\{-c_{0}(E, N) \sqrt{\log x}\right\} .
$$

The proof of this proposition relies heavily on the following, the content of which is drawn from Propositions 3.4 and 3.8 of [15, pp. 255, 258].

Lemma 1. Let $F / E$ be an Abelian extension of number fields, let $\eta$ be a character of $\operatorname{Gal}(F / E)$, and let $\mathcal{L}(t):=\frac{1}{2} \log A(\eta)+n_{E} \log (|t|+2)$. There is an absolute positive constant $c_{1}$ such that $L(s, \eta)$ has at most one zero $\sigma+i t$ in the region

$$
1-\frac{c_{1}}{\mathcal{L}(t)} \leq \sigma \leq 1
$$

If such a zero exists, then it is real and simple, and $\eta$ must be a character of order dividing 2. Furthermore, we refer to such a zero as an exceptional zero of $L(s, \eta)$ and denote it by $\beta_{\eta}$.

Let $\epsilon>0$, and let $\tilde{E}$ denote the normal closure of $E$ over $\mathbb{Q}$. If $\eta$ is a character of $\operatorname{Gal}(F / E)$ for which $\beta_{\eta}$ exists, then there is a positive constant $c_{2}(\epsilon)$ so that

$$
\beta_{\eta} \leq \max \left\{1-\frac{c_{2}(\epsilon)}{\left(\left|d_{\tilde{E}}\right|^{2} A(\eta)\right)^{\epsilon}}, 1-\frac{1}{16 n_{\tilde{E}} \log \left(\left|d_{\tilde{E}}\right|^{2} A(\eta)\right)}\right\} .
$$

Remark. We observe that if $\eta$ is a character for which $\beta_{\eta}$ exists and $\beta_{\eta}<1 / 2$, then the functional equation for $L(s, \eta)$ implies that $1-\beta_{\eta}$ is also a zero since $\eta=\bar{\eta}$. In this case, we would have

$$
1-\frac{c_{1}}{\mathcal{L}(0)} \leq \beta_{\eta}<\frac{1}{2}<1-\beta_{\eta}
$$


which contradicts Lemma 1. Thus, we may assume that if $\beta_{\eta}$ exists, then

$$
\frac{1}{2} \leq 1-\frac{c_{1}}{\mathcal{L}(0)} \leq \beta_{\eta}
$$

Remark. Applying Lemma 1 to the character $\bar{\eta}$ and appealing to the functional equation for $L(s, \eta)$, we see that $L(s, \eta)$ has at most one zero $\sigma+i t$ in the region

$$
0 \leq \sigma \leq \frac{c_{1}}{\mathcal{L}(t)}
$$

Furthermore, if such a zero exists, it must be simple, $\eta=\bar{\eta}$ must be a character of order dividing 2 , and we deduce that this zero equal to $1-\beta_{\eta}$.

Remark. The second part of Lemma 1 depends on Siegel's Theorem [4, p. 126], and as such is ineffective. This, of course, means that Proposition 1 is ineffective as well.

Proof of Proposition [1. The proof follows the traditional method as in [10]. For convenience, we write $\eta=\omega \otimes \chi$. We begin with integral identity

$$
\widetilde{\psi}\left(x ; L\left(\zeta_{q}\right) / E, \eta\right)=-\frac{1}{2 \pi i} \int_{(2)} \frac{L^{\prime}(s, \eta)}{L(s, \eta)} \frac{x^{s}}{s} d s .
$$

With $\sigma_{0}=1+(\log x)^{-1}$ and $T$ a real parameter, we estimate the right-hand side by the truncated integral

$$
I_{\eta}(x, T):=-\frac{1}{2 \pi i} \int_{\sigma_{0}-i T}^{\sigma_{0}+i T} \frac{L^{\prime}(s, \eta)}{L(s, \eta)} \frac{x^{s}}{s} d s .
$$

We note that our definition of $I_{\eta}(x, T)$ differs from that in [10, p. 440] by a negative sign.

Arguing as in [10, pp. 424-428], for $x \geq 2$ and $T>0$, we have

$$
\widetilde{\psi}\left(x ; L\left(\zeta_{q}\right) / E, \eta\right)-I_{\eta}(x, T) \ll n_{E} \log x+\frac{n_{E} x(\log x)^{2}}{T},
$$

where the implied constant is absolute. Since $\chi$ is assumed to be nontrivial, it follows that $\eta=\omega \otimes \chi$ is as well. Now, provided that $x, T \geq 2$ and that $T$ does not coincide with the imaginary part of any zero of $L\left(s ; L\left(\zeta_{q}\right) / E, \eta\right)$, in [10, p.450], we find that

$$
I_{\eta}(x, T)+\sum_{\substack{\rho=\beta+i \gamma \\|\gamma|<T}} \frac{x^{\rho}}{\rho}-\sum_{\substack{\rho=\beta+i \gamma \\|\rho|<\frac{1}{2}}} \frac{1}{\rho} \ll \log A(\eta)+n_{E} \log x+\frac{x \log x}{T}\left(\log A(\eta)+n_{E} \log T\right),
$$

where both sums are taken over the nontrivial zeros $\rho$ of $L\left(s ; L\left(\zeta_{q}\right) / E, \eta\right)$ and the implied constant is absolute. If, however, $T$ does coincide with the imaginary part of some zero $\rho$, we may argue as in [10, p.451] and correct the problem (by simply increasing the constant implied by the $\ll$ notation). then

By Lemma 1, if $\rho=\beta+i \gamma \neq \beta_{\eta}$ is not an exceptional zero of $L\left(s ; L\left(\zeta_{q}\right) / E, \eta\right)$ and $|\gamma|<T$,

$$
\left|x^{\rho}\right| \leq x \exp \left\{-\frac{c_{1} \log x}{\mathcal{L}(T)}\right\}
$$

Let $N_{\eta}(t)$ denote the number of nontrivial zeros $\rho=\beta+i \gamma$ of $L\left(s ; L\left(\zeta_{q}\right) / E, \eta\right)$ satisfying the condition $|\gamma-t| \leq 1$. By Lemma 5.4 of [10, p. 436], $N_{\eta}(t) \ll \log A(\eta)+n_{E} \log (|t|+2)$. 
Thus,

$$
\begin{aligned}
\sum_{\substack{\rho=\beta+i \gamma \\
\rho \neq \beta_{\eta} \\
|\rho| \geq 1 / 2 \\
|\gamma|<T}}\left|\frac{x^{\rho}}{\rho}\right| & \ll x \exp \left\{-\frac{c_{1} \log x}{\mathcal{L}(T)}\right\} \sum_{j<T} \frac{N_{\eta}(j)}{j} \\
& \ll x \exp \left\{-\frac{c_{1} \log x}{\mathcal{L}(T)}\right\}\left(\log A(\eta)+n_{E} \log T\right) \log T,
\end{aligned}
$$

the implied constants being absolute. By the second remark following Lemma 1, if $\rho=$ $\beta+i \gamma \neq 1-\beta_{\eta}$, then $\beta \geq \frac{c_{1}}{\mathcal{L}(t)}$ and hence

$$
\frac{1}{|\rho|} \leq \frac{\mathcal{L}(\gamma)}{c_{1}} \ll \log A(\eta)+n_{E},
$$

the implied constant being absolute. Thus,

$$
\begin{aligned}
\sum_{\substack{\rho=\beta+i \gamma \\
\rho \neq 1-\beta_{\eta} \\
|\rho|<\frac{1}{2}}}\left|\frac{x^{\rho}}{\rho}\right|+\left|\frac{1}{\rho}\right| & \ll x^{1 / 2} \sum_{\substack{|\rho|<\frac{1}{2} \\
\rho \neq 1-\beta_{\eta}}} \frac{1}{|\rho|} \ll x^{1 / 2} N_{\eta}(0) \max _{\substack{|\rho|<\frac{1}{2} \\
\rho \neq 1-\beta_{\eta}}}\left\{\frac{1}{|\rho|}\right\} \\
& \ll x^{1 / 2}\left(\log A(\eta)+n_{E}\right)^{2},
\end{aligned}
$$

where again, all implied constants are absolute. By the Mean Value Theorem,

$$
\frac{x^{1-\beta_{\eta}}}{1-\beta_{\eta}}-\frac{1}{1-\beta_{\eta}}=\frac{x^{1-\beta_{\eta}}-1}{1-\beta_{\eta}}=x^{\sigma} \log x
$$

for some $0<\sigma<1-\beta_{\eta} \leq \frac{1}{2}$. By the second part of Lemma 1, there exists a positive constant $c_{3}(E, \epsilon)$ depending on $E$ and $\epsilon$ so that

$$
\frac{1}{2} \leq \beta_{\eta} \leq 1-\frac{c_{3}(E, \epsilon)}{A(\eta)^{\epsilon}}
$$

Hence,

$$
\left|\frac{x^{\beta_{\eta}}}{\beta_{\eta}}\right| \leq 2 x^{\beta_{\eta}} \leq 2 x \exp \left\{-c_{3}(E, \epsilon) \frac{\log x}{A(\eta)^{\epsilon}}\right\} \ll x \exp \left\{-c_{3}(E, \epsilon)(\log x)^{1-N \epsilon}\right\},
$$

where the implied constant is absolute. Therefore,

$$
\begin{gathered}
\sum_{\substack{\rho=\beta+i \gamma \\
|\gamma|<T}} \frac{x^{\rho}}{\rho}-\sum_{\substack{\rho=\beta+i \gamma \\
|\rho|<\frac{1}{2}}} \frac{1}{\rho} \ll \\
+x \exp \left\{-c_{1} \frac{\log x}{\mathcal{L}(T)}\right\}(\log A(\eta)+\log T) \log T+\sqrt{x}\left(\log A(\eta)+n_{E}\right)^{2} \\
\left.+-c_{3}(E, \epsilon)(\log x)^{1-N \epsilon}\right\}+\sqrt{x}(\log x)
\end{gathered}
$$

where the implied constant is absolute. Choosing $\epsilon=1 / 2 N$,

$$
T=\exp \left\{\frac{1}{n_{E}}\left(\sqrt{\log x}-\frac{1}{2} \log A(\eta)\right)\right\},
$$

and combining (11), (12), and (13), the proposition follows. 
We now return to the situation described in the introduction. That is, we let $L / K$ be a Galois (not necessarily Abelian) extension of number fields with group $G$. Further, we let $C$ be a fixed conjugacy class in $G$, and we let $\delta_{C}$ denote the characteristic function of $C$. Given a Dirichlet character $\chi$ modulo $q$, we now wish to approximate

$$
\theta\left(x ; \delta_{C} \otimes \chi\right)=\sum_{\substack{\mathbf{N} \mathfrak{p} \leq x \\\left(\frac{L / K}{\mathfrak{p}}\right)=C}} \chi(\mathbf{N p}) \log \mathbf{N p} .
$$

In the proofs, it will be more convenient to work with $\widetilde{\psi}$ instead of $\theta$. By (8), we have

$$
\left|\widetilde{\psi}\left(x ; L\left(\zeta_{q}\right) / K, \delta_{C} \otimes \chi\right)-\theta\left(x ; \delta_{C} \otimes \chi\right)\right| \leq n_{K}\left(\sqrt{x} \log x+\frac{2}{\varphi_{K}(q) n_{L}} \log \left|d_{L\left(\zeta_{q}\right)}\right|\right)
$$

as $n_{L\left(\zeta_{q}\right)}=\varphi_{K}(q) n_{L}$. Since $\left[L\left(\zeta_{q}\right): \mathbb{Q}\left(\zeta_{q}\right)\right]=\varphi_{K}(q) n_{L} / \varphi(q)$, by Lemma 7 of [22, p. 143], we have $\left|d_{L\left(\zeta_{q}\right)}\right| \leq\left|d_{L}\right|^{\varphi_{K}(q)} \cdot\left|d_{\mathbb{Q}\left(\zeta_{q}\right)}\right|^{n_{L} \varphi_{K}(q) / \varphi(q)}$. In [23, p. 12], we find the identity

$$
d_{\mathbb{Q}\left(\zeta_{q}\right)}=(-1)^{\varphi(q) / 2} \frac{q^{\varphi(q)}}{\prod_{\ell \mid q} \ell^{\varphi(q) /(\ell-1)}},
$$

where the product is over the distinct primes $\ell$ dividing $q$. Thus, we have

$$
\frac{\log \left|d_{L\left(\zeta_{q}\right)}\right|}{\varphi_{K}(q) n_{L}} \leq \frac{\log \left|d_{L}\right|}{n_{L}}+\log q
$$

and therefore,

$$
\left|\theta\left(x ; \delta_{C} \otimes \chi\right)-\widetilde{\psi}\left(x ; L\left(\zeta_{q}\right) / K, \delta_{C} \otimes \chi\right)\right| \leq n_{K}\left(\sqrt{x} \log x+\frac{2}{n_{L}} \log \left|d_{L}\right|+2 \log q\right) .
$$

We recall that the Galois group $\operatorname{Gal}\left(K\left(\zeta_{q}\right) / K\right)$ is isomorphic to some subgroup of $(\mathbb{Z} / q \mathbb{Z})^{*}$, which we denote by $G_{K, q}$. Now, let $G_{K, q}^{\perp}$ denote the subgroup of Dirichlet characters modulo $q$ that are trivial on $G_{K, q}$. Then two Dirichlet characters $\chi_{1}, \chi_{2}$ define the same map on $G_{K, q}$ whenever $\chi_{1} \equiv \chi_{2}\left(\bmod G_{K, q}^{\perp}\right)$. Throughout, we will reserve the notation $\chi_{0}$ for the trivial Dirichlet character modulo $q$. That is, $\chi_{0}(a)=1$ if $(a, q)=1$ and $\chi_{0}(a)=0$ otherwise.

Lemma 2. There exists a positive constant $c_{4}(L)$ so that for any Dirichlet character modulo $q \leq x$ with $\chi \equiv \chi_{0}\left(\bmod G_{K, q}^{\perp}\right)$, we have

$$
\theta\left(x ; \delta_{C} \otimes \chi\right)-\frac{|C|}{|G|} x \ll x \exp \left\{-c_{4}(L) \sqrt{\log x}\right\},
$$

where the implied constant depends only on $L$.

Proof. Properties of $L$-functions imply that

$$
\widetilde{\psi}\left(x ; L\left(\zeta_{q}\right) / K, \delta_{C} \otimes \chi\right)=\widetilde{\psi}\left(x ; L\left(\zeta_{q}\right) / K, \delta_{C} \otimes \chi_{0}\right)=\widetilde{\psi}\left(x ; L / K, \delta_{C}\right) .
$$

By the Effective Chebotarëv Density Theorem [10, p. 458], we obtain

$$
\widetilde{\psi}\left(x ; L / K, \delta_{C}\right)=\frac{|C|}{|G|} x-\frac{|C|}{|G|} \frac{x^{\beta_{0}}}{\beta_{0}}+O\left(x \exp \left\{-c_{5} n_{L}^{-1 / 2} \sqrt{\log x}\right\}\right),
$$

where $c_{5}$ is an absolute positive constant, $\beta_{0}$ is a potential "exceptional zero" of the Dedekind zeta function for $L$, and the convention is that the term involving $\beta_{0}$ should be removed if 
$\beta_{0}$ does not exist. Furthermore, the constant implied by the big- $O$ is absolute. See [10, pp. 413-414] for the precise definition of $\beta_{0}$. Stark's bound gives

$$
\beta_{0}<\max \left\{1-1 /\left(4 \log \left|d_{L}\right|\right), 1-c_{6} /\left|d_{L}\right|^{1 / n_{L}}\right\} .
$$

See [15, p. 249] or [22, p. 148] for example. Therefore,

$$
\widetilde{\psi}\left(x ; L / K, \delta_{C}\right)=\frac{|C|}{|G|} x+O\left(x \exp \left\{-c_{7} n_{L}^{-1 / 2} \sqrt{\log x}\right\}\right),
$$

where the implied constant depends on $L$. The result now follows by (14).

We now turn those characters $\chi \not \equiv \chi_{0}\left(\bmod G_{K, q}^{\perp}\right)$. For $q$ smaller than a power of $\log x$, we apply Proposition 1 and the technique of Deuring [6] and Macluer [13] to obtain good bounds for $\theta\left(x ; \delta_{C} \otimes \chi\right)$.

Lemma 3. Let $M>0$ and $q \leq(\log x)^{M}$. There exists a positive constant $c_{8}(L, M)$ so that if $\chi \not \equiv \chi_{0}\left(\bmod G_{K, q}^{\perp}\right)$, then

$$
\theta\left(x ; \delta_{C} \otimes \chi\right) \ll x \exp \left\{-c_{8}(L, M) \sqrt{\log x}\right\},
$$

where the implied constant depends only on L.

Proof. Ideally, one would like to begin by decomposing $\delta_{C}$ as a linear combination of irreducible characters $\eta$ of $G$ and then obtain good bounds for $\left|\widetilde{\psi}\left(x ; L\left(\zeta_{q}\right) / K, \eta \otimes \chi\right)\right|$. However, this is complicated by the fact that $\eta$ and hence $\eta \otimes \chi$ may be associated to a representation $\rho$ of $\operatorname{Gal}\left(L\left(\zeta_{q}\right) / K\right)$ of dimension greater than one. In fact, unless the extension $L / K$ is Abelian, there will always be at least one such $\eta$. See [18, p. 25] for example.

We instead employ the argument of Deuring [6] and MacCluer [13] to reduce to the case of one dimensional characters. See also [15, pp. 246, 250] and [10, pp. 429-430]. In particular, we choose some Abelian subgroup $H$ of $G$ so that $H \cap C \neq\{\}$. Note that if $g_{C} \in C$, then $H=\left\langle g_{C}\right\rangle$ is one possible choice. Now, take $h_{C} \in H \cap C$, and let $C_{H}$ denote the conjugacy class of $h_{C}$ in $H$. Then

$$
\delta_{C}=\frac{|C| \cdot|H|}{|G| \cdot\left|C_{H}\right|} \operatorname{Ind}_{H}^{G} \delta_{C_{H}} .
$$

Put $\mathcal{H}_{q}:=H \times G_{K, q}$. By Mackey's Induction Theorem [18, p. 57], we have

$$
\delta_{C} \otimes \chi=\frac{|C| \cdot|H|}{|G| \cdot\left|C_{H}\right|} \operatorname{Ind}_{\mathcal{H}_{q}}^{\mathcal{G}_{q}}\left(\delta_{C_{H}} \otimes \chi\right),
$$

where we are using the same symbol $\chi$ to represent the character on $\mathcal{G}_{q}$ and the associated character on $\mathcal{H}_{q}$. Now, let $E$ be the subfield of $L\left(\zeta_{q}\right)$ fixed by $\mathcal{H}_{q}$, and note that $E$ does not depend on $q$ since it is also the subfield of $L$ fixed by $H$. By the invariance of $L$-functions under induction, we have

$$
\begin{aligned}
\widetilde{\psi}\left(x ; L\left(\zeta_{q}\right) / K, \delta_{C} \otimes \chi\right) & =\frac{|C| \cdot|H|}{|G| \cdot\left|C_{H}\right|} \widetilde{\psi}\left(x ; L\left(\zeta_{q}\right) / K, \operatorname{Ind}_{\mathcal{H}_{q}}^{\mathcal{G}_{q}}\left(\delta_{C_{H}} \otimes \chi\right)\right) \\
& =\frac{|C| \cdot|H|}{|G| \cdot\left|C_{H}\right|} \widetilde{\psi}\left(x ; L\left(\zeta_{q}\right) / E, \delta_{C_{H}} \otimes \chi\right) .
\end{aligned}
$$


Now we may write

$$
\delta_{C_{H}}=\frac{\left|C_{H}\right|}{|H|} \sum_{\omega} \bar{\omega}\left(h_{C}\right) \omega,
$$

where $\omega$ ranges over all the irreducible (one-dimensional) characters of $H$. Thus,

$$
\left|\widetilde{\psi}\left(x ; L\left(\zeta_{q}\right) / K, \delta_{C} \otimes \chi\right)\right| \leq \frac{|C|}{|G|} \sum_{\omega}\left|\widetilde{\psi}\left(x ; L\left(\zeta_{q}\right) / E, \omega \otimes \chi\right)\right| .
$$

Since $\chi \not \equiv \chi_{0}\left(\bmod G_{K, q}^{\perp}\right)$, it follows that $\omega \otimes \chi$ is not trivial. By (10) and the conductordiscriminant formula,

$$
\sum_{\omega} \log A(\omega \otimes \chi) \leq \log \left(\left|d_{L}\right| q^{n_{L}}\right)
$$

The result now follows by applying Proposition 1 to (17) and using (14).

\section{Proof of Theorem 1}

Given two Dirichlet characters, say $\chi_{1}$ and $\chi_{2}$, it follows that $\theta\left(x ; \delta_{C} \otimes \chi_{1}\right)=\theta\left(x ; \delta_{C} \otimes \chi_{2}\right)$ if $\chi_{1} \equiv \chi_{2}\left(\bmod G_{K, q}^{\perp}\right)$. Therefore, we set

$$
E\left(x ; \delta_{C} \otimes \chi\right):= \begin{cases}\theta\left(x ; \delta_{C} \otimes \chi\right)-\frac{|C|}{|G|} x & \text { if } \chi \equiv \chi_{0} \quad\left(\bmod G_{K, q}^{\perp}\right), \\ \theta\left(x ; \delta_{C} \otimes \chi\right) & \text { otherwise. }\end{cases}
$$

For each $q$, let $\widehat{G}_{K, q}$ denote a complete set of coset representatives for the quotient group of Dirichlet characters modulo $G_{K, q}^{\perp}$. We begin with the decomposition

$$
\theta(x ; C, q, a)-\frac{|C|}{\varphi_{K}(q)|G|} x=\frac{1}{\varphi_{K}(q)} \sum_{\chi \in \widehat{G_{K, q}}} \bar{\chi}(a) E\left(x ; \delta_{C} \otimes \chi\right) .
$$

Then squaring, summing over $a \in G_{K, q}$, and applying orthogonality relations as in [4, p. 170] or [20, p. 2740], we have

$$
\begin{aligned}
\sum_{a \in G_{K, q}}\left|\theta(x ; C, q, a)-\frac{|C|}{\varphi_{K}(q)|G|} x\right|^{2} & =\frac{1}{\varphi_{K}(q)^{2}} \sum_{a \in G_{K, q}}\left|\sum_{\chi \in \widehat{G_{K}, q}} \bar{\chi}(a) E\left(x ; \delta_{C} \otimes \chi\right)\right|^{2} \\
& =\frac{1}{\varphi_{K}(q)} \sum_{\chi \in \widehat{G_{K, q}}}\left|E\left(x ; \delta_{C} \otimes \chi\right)\right|^{2} \\
& =\frac{1}{\varphi(q)} \sum_{\chi \bmod q}\left|E\left(x ; \delta_{C} \otimes \chi\right)\right|^{2} .
\end{aligned}
$$

In the last line above, the sum is taken over all Dirichlet characters modulo $q$.

For each Dirichlet character $\chi$, we let $\chi_{*}$ denote the primitive character that induces $\chi$. Summing the above over only those $q$ such that $L \cap K\left(\zeta_{q}\right)=K$ and replacing each character $\chi$ by the primitive character that induces it, we find that

$$
\sum_{q \leq Q}^{\prime} \sum_{a \in G_{K, q}}\left(\theta(x ; C, q, a)-\frac{1}{\varphi_{K}(q)} \frac{|C|}{|G|} x\right)^{2} \ll \sum_{q \leq Q}^{\prime}(\log q)^{2}+\sum_{q \leq Q}^{\prime} \frac{1}{\varphi(q)} \sum_{\chi \bmod q}\left|E\left(x ; \delta_{C} \otimes \chi_{*}\right)\right|^{2} .
$$


Since $\sum_{q \leq Q}(\log q)^{2} \leq Q(\log Q)^{2}<x Q \log x$, we concentrate on the second sum on the right. Arguing as in [4, p. 170], we note that if $\chi$ is primitive modulo $q$, then $\chi$ only induces characters to moduli which are multiples of $q$. Hence,

$$
\sum_{q \leq Q}^{\prime} \frac{1}{\varphi(q)} \sum_{\chi \in \mathcal{X}(q)}\left|E\left(x ; \delta_{C} \otimes \chi_{*}\right)\right|^{2}=\sum_{q \leq Q}^{\prime} \sum_{\chi}^{*}\left|E\left(x ; \delta_{C} \otimes \chi\right)\right|^{2} \sum_{k \leq Q / q} \frac{1}{\varphi(k q)},
$$

where the ${ }^{*}$ on the sum over $\chi$ denotes the fact that the sum is to be restricted to those $\chi$ which are primitive modulo $q$. Since $\sum_{k \leq Q / q} \frac{1}{\varphi(k q)} \ll \varphi(q)^{-1} \log (2 Q / q)$,

$$
\sum_{q \leq Q}^{\prime} \frac{1}{\varphi(q)} \sum_{\chi \in \mathcal{X}(q)}\left|E\left(x ; \delta_{C} \otimes \chi_{*}\right)\right|^{2} \ll \sum_{q \leq Q}^{\prime} \frac{1}{\varphi(q)} \log (2 Q / q) \sum_{\chi}^{*}\left|E\left(x ; \delta_{C} \otimes \chi\right)\right|^{2} ;
$$

and we see that Theorem 1 follows from the following proposition.

Proposition 2. Let $M>0$. If $x(\log x)^{-M} \leq Q \leq x$, then

$$
\sum_{q \leq Q}{ }^{\prime} \frac{1}{\varphi(q)} \log (2 Q / q) \sum_{\chi}{ }^{*}\left|E\left(x ; \delta_{C} \otimes \chi\right)\right|^{2} \ll x Q \log x
$$

where the' on the outer sum indicates that the sum is to be restricted to those $q$ such that $L \cap K\left(\zeta_{q}\right)=K$ and the ${ }^{*}$ on the inner sum indicates that the sum is to be restricted to those characters $\chi$ which are primitive modulo $q$. The implied constant depends on $L$ and $K$.

In order to prove Proposition 2, we divide the outer sum over $q$ into two groupings depending on the size of $q$. The "large" $q$ are handled via the large sieve while the "small" $q$ are handled via the results in Section 4. For the convenience of the reader, we give a statement of the large sieve inequality as found in [9, p. 179].

Theorem 3 (Large sieve inequality). For any complex numbers $a_{n}$ with $N_{0}<n \leq N_{0}+N$, where $N$ is a positive integer, we have

$$
\sum_{q \leq Q} \frac{q}{\varphi(q)} \sum_{\chi}^{*}\left|\sum_{N_{0}<n \leq N_{0}+N} \chi(n) a_{n}\right|^{2} \leq\left(Q^{2}+N-1\right) \sum_{N_{0}<n \leq N_{0}+N}\left|a_{n}\right|^{2} .
$$

Proof of Proposition [. Fix $M>0$, and put $Q_{1}:=(\log x)^{M+1}$. Applying the large sieve, we obtain

$$
\sum_{q \leq Q}^{\prime} \frac{q}{\varphi(q)} \sum_{\chi}^{*}\left|\theta\left(x ; \delta_{C} \otimes \chi\right)\right|^{2} \leq n_{K}\left(Q^{2}+x\right) \sum_{\substack{\mathbf{N} \mathfrak{p} \leq x \\\left(\frac{L / K}{\mathfrak{p}}\right)=C}}(\log \mathbf{N p})^{2} \ll\left(Q^{2}+x\right) x \log x,
$$

where the implied constant depends on $L$. Thus, we have

$$
\sum_{U<q \leq 2 U}{ }^{\prime} \frac{1}{\varphi(q)} \sum_{\chi}^{*}\left|\theta\left(x ; \delta_{C} \otimes \chi\right)\right|^{2} \ll x \log x\left(U+x U^{-1}\right) \log (2 Q / U) .
$$

Summing over $U=Q 2^{-k}$, for $Q$ as specified in the statement of Proposition 2, we have

$$
\begin{aligned}
\sum_{Q_{1}<q \leq Q}{ }^{\prime} \frac{1}{\varphi(q)} \log (2 Q / q) \sum_{\chi}^{*}\left|\theta\left(x ; \delta_{C} \otimes \chi\right)\right|^{2} & \ll x^{2} Q_{1}^{-1}(\log x)^{2}+x Q \log x \\
& \ll x Q \log x,
\end{aligned}
$$


where the implied constant depends on $L$. We note that $E\left(x ; \delta_{C} \otimes \chi\right) \ll \theta\left(x ; \delta_{C} \otimes \chi\right)$ by Lemma 2 and Lemma 3. Hence,

$$
\sum_{Q_{1}<q \leq Q}{ }^{\prime} \frac{1}{\varphi(q)} \log (2 Q / q) \sum_{\chi}^{*}\left|E\left(x ; \delta_{C} \otimes \chi\right)\right|^{2} \ll x Q \log x,
$$

where the implied constant depends on $L$. This handles the large values of $q$.

For the contribution arising from the small values of $q$, viz., $q \leq Q_{1}=(\log x)^{M+1}$, we apply Lemmas 2 and 3 to obtain the bound

$$
\begin{aligned}
\sum_{q \leq Q_{1}}{ }^{\prime} \frac{1}{\varphi(q)} \log (2 Q / q) \sum_{\chi}{ }^{*}\left|E\left(x ; \delta_{C} \otimes \chi\right)\right|^{2} & \ll Q_{1}(\log Q) x^{2} \exp \{-c(L, M) \sqrt{\log x}\} \\
& \ll x^{2}(\log x)^{-M} \ll x Q \log x,
\end{aligned}
$$

where the implied constant depends on $L$ and $M$.

\section{REFERENCES}

[1] M.B. Barban. On the distribution of primes in arithmetic progressions "on average". Dokl. Akad. Nauk UzSSR, 5:5-7, 1964. (Russian).

[2] H. Davenport and H. Halberstam. Primes in arithmetic progressions. Michigan Math. J., 13:485-489, 1966.

[3] H. Davenport and H. Halberstam. Corrigendum: "Primes in arithmetic progression". Michigan Math. $J ., 15: 505,1968$.

[4] Harold Davenport. Multiplicative Number Theory. Springer-Verlag, New York, 1980.

[5] Chantal David and Francesco Pappalardi. Average Frobenius distribution for inerts in $\mathbb{Q}(i)$. J. Ramanujan Math. Soc., 19(3):181-201, 2004.

[6] Max Deuring. Über den Tschebotareffschen Dichtigkeitssatz. Math. Ann., 110(1):414-415, 1935.

[7] Bryan Faulkner, Kevin James, Matthew King, and David Penniston. Average Frobenius distributions for elliptic curves over Abelian extensions. Acta Arith. (to appear).

[8] Christopher Hooley. On the Barban-Davenport-Halberstam theorem. I. J. Reine Angew. Math., 274/275:206-223, 1975. Collection of articles dedicated to Helmut Hasse on his seventy-fifth birthday, III.

[9] Henryk Iwaniec and Emmanuel Kowalski. Analytic Number Theory, volume 53 of American Mathematical Society Colloquium Publications. American Mathematical Society, Providence, RI, 2004.

[10] J. C. Lagarias and A. M. Odlyzko. Effective versions of the Chebotarev density theorem. In Algebraic number fields: L-functions and Galois properties (Proc. Sympos., Univ. Durham, Durham, 1975), pages 409-464. Academic Press, London, 1977.

[11] Serge Lang. Algebraic Number Theory, volume 110 of Graduate Texts in Mathematics. Springer-Verlag, New York, second edition, 1994.

[12] Serge Lang. Algebra. Springer-Verlag, New York, 3 edition, 2002.

[13] C. R. MacCluer. A reduction of the Cebotarev density theorem to the cyclic case. Acta Arith., 15:45-47, 1968.

[14] J. Martinet. Character theory and Artin L-functions. In Algebraic number fields: L-functions and Galois properties (Proc. Sympos., Univ. Durham, Durham, 1975), pages 1-87. Academic Press, London, 1977.

[15] M. Ram Murty and V. Kumar Murty. A variant of the Bombieri-Vinogradov theorem. In Number theory (Montreal, Que., 1985), volume 7 of CMS Conf. Proc., pages 243-272. Amer. Math. Soc., Providence, RI, 1987.

[16] M. Ram Murty and V. Kumar Murty. Non-vanishing of L-functions and Applications, volume 157 of Progress in Mathematics. Birkhäuser Verlag, Basel, 1997.

[17] M. Ram Murty and Kathleen L. Petersen. A Bombieri-Vinogradov theorem for all number fields. (preprint). 
[18] Jean-Pierre Serre. Linear Representations of Finite Groups. Springer-Verlag, New York, 1977. Translated from the second French edition by Leonard L. Scott, Graduate Texts in Mathematics, Vol. 42.

[19] Jean-Pierre Serre. Quelques applications du théorème de densité de Chebotarev. Inst. Hautes Études Sci. Publ. Math., (54):323-401, 1981.

[20] Ethan Smith. A generalization of the Barban-Davenport-Halberstam Theorem to number fields. $J$. Number Theory, 129(11):2735-2742, 2009.

[21] Ethan Smith. A Barban-Davenport-Halberstam asymptotic for number fields. Proc. Amer. Math. Soc., 138(7):2301-2309, 2010.

[22] H. M. Stark. Some effective cases of the Brauer-Siegel theorem. Invent. Math., 23:135-152, 1974.

[23] Lawrence C. Washington. Introduction to Cyclotomic Fields, volume 83 of Graduate Texts in Mathematics. Springer-Verlag, New York, second edition, 1997.

Centre de recherches mathématiques, Université de Montréal, P.O. Box 6128, Centreville Station, Montréal, Québec, H3C 3J7, Canada; and Department of Mathematical Sciences, Michigan Technological University, 1400 Townsend Drive, Houghton, Michigan, 49931-1295, USA

E-mail address: ethans@mtu.edu

$U R L$ : www.math.mtu.edu/ ${ }^{\sim}$ ethans 